\section{Reacción de hipersensibilidad secundaria al uso de bloqueadores neuromusculares}

\author{
Hypersensitivity reaction secondary to the use of neuromuscular blockers \\ Dr. Rodner Ernesto Fuentes-Broqueet,* Prof. Dra. Idoris Cordero-Escobar, ${ }^{*}$ \\ Dra. Gisela Pérez-Martínez, ${ }^{\S}$ Dra. Isabel Mora-Díaz
}

Citar como: Fuentes-Broqueet RE, Cordero-Escobar I, Pérez-Martínez G, Mora-Díaz I. Reacción de hipersensibilidad secundaria al uso de bloqueadores neuromusculares. Rev Mex Anestesiol. 2021; 44 (2): 105-109. https://dx.doi. $\operatorname{org} / 10.35366 / 99013$

RESUMEN. Introducción: La anafilaxia perioperatoria constituye una condición clínica potencialmente letal. La causa más frecuente se atribuye a los bloqueadores neuromusculares. Objetivo: Identificar la incidencia de reacciones anafilácticas secundarias al uso de bloqueadores neuromusculares. Material y métodos: Se realizó una investigación descriptiva, observacional, de corte transversal para evaluar la incidencia de reacciones anafilácticas secundarias al uso de bloqueantes neuromusculares. El estudio se realizó en el Hospital «Hermanos Ameijeiras» en el período comprendido entre enero de 2016 y diciembre de 2018. Resultados: Del total de intervenciones quirúrgicas electivas, 3,431 requirieron anestesia general y el uso de bloqueadores neuromusculares. Predominó el sexo femenino en $75 \%$ de los casos, el grupo etario de 60 años y más con 68 pacientes (32.7\%), el estado físico ASA II, 98 pacientes (41.1\%). La media del IMC fue de $22.7 \pm 1.14$. La media del tiempo quirúrgico fue de $190 \pm 42.5 \mathrm{~min}$. De todos los fármacos el más utilizado fue el atracurio en 90 pacientes $(43.3 \%)$ seguido del vecuronio $79(38.0 \%)$ y el rocuronio $39(18.8 \%)$. El número de eventos adversos fue escaso. Sólo se encontraron cuatro, dos con atracurio $(50 \%)$, uno con rocuronio y uno con vecuronio $25 \%$ respectivamente. Conclusiones: Se constató la presencia de reacciones anafilácticas por el uso de bloqueadores neuromusculares, mismas que se manifestaron en un corto período al inicio de la inducción. El atracurio presentó la mayor frecuencia y todas fueron de intensidad leve.

ABSTRACT. Introduction: Perioperative anaphylaxis is a potentially lethal clinical condition. The most frequent cause is attributed to neuromuscular blockers. Objective: To identify the incidence of anaphylactic reactions secondary to the use of neuromuscular blockers. Material and methods: A descriptive, observational, cross-sectional investigation was conducted to assess the incidence of anaphylactic reactions secondary to the use of neuromuscular blockers. The study was carried out at the «Hermanos Ameijeiras» Hospital, in the period between january 2016 and december 2018. Results: Of the total elective surgical interventions, 3,431 required general anesthesia and the use of neuromuscular blockers. The female sex predominated with $75 \%$, the age group of 60 years and over with 68 patients (32.7\%), ASA II physical condition, 98 patients (41.1\%). The average BMI was $22.7 \pm 1.14$. The mean surgical time was $190 \pm 42.5$ min. Of all the drugs the most used was atracurium in 90 patients (43.3\%), followed by vecuronium 79 (38.0\%) and rocuronium 39 (18.8\%). The number of adverse events was low. Only four were found, two with atracurium (50\%), one with rocuronium and one with $25 \%$ vecuronium respectively. Conclusions: The presence of anaphylactic reactions was observed with the use of neuromuscular blockers, which occurred in a short period at the beginning of induction. The atracurium presented the highest frequency and all were of mild intensity.

\section{INTRODUCCIÓN}

D urante el acto anestésico quirúrgico, la anafilaxia perioperatoria constituye una condición clínica potencialmente mortal que suele ser el resultado del empleo de medicamentos o sustancias que se utilizan durante el acto anestésico-quirúrgico. La causa más frecuente se atribuye a los bloqueadores neuromusculares (BNM) $)^{(1-5)}$.

Liew y colaboradores ${ }^{(4)}$ definieron la anafilaxia como una reacción alérgica grave que tiene un inicio rápido y puede causar la muerte. Estos autores publicaron que la tasa varía entre los diferentes países a partir de 1/10,000 a 1/20,000.

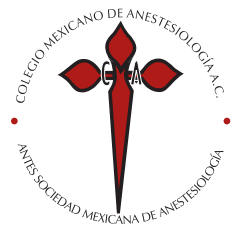

Palabras clave: Reacciones anafilácticas perioperatorias, complicaciones, bloqueadores neuromusculares.

Keywords:

Perioperative anaphylactic reactions, complications, neuromuscular blockers.

* Especialista en Anestesiología

y Reanimación. Servicio de Anestesiología y Reanimación. ORCID. http://orcid.org/00000003-1359-6854.

‡ Especialista en Anestesiología y Reanimación. DCs. Profesora e Investigadora Titular. Servicio de Anestesiología y Reanimación. ORCID. http:// orcid.org/0000-0001-9877-3113. $\S$ Especialista en Anestesiología y Reanimación. Profesora Auxiliar. Servicio de Anestesiología y Reanimación. ORCID. ORCID. http://orcid. org/0000-0001-7428-1165.

^ Especialista en Bioestadística. MSc. en Urgencias Médicas. Profesora Asistente. Máster en Servicio de Investigaciones. ORCID. http:// orcid.org/0000-0002-8491-7945.

Hospital Clínico Quirúrgico «Hermanos Ameijeiras». La Habana, Cuba

Correspondencia: Prof. Dra. Idoris Cordero-Escobar E-mail: ice@infomed.sld.cu

Recibido: 15-09-2019

Aceptado: 05-01-2020 
La anafilaxia es la causa más común de complicaciones durante la anestesia, independiente del proceder quirúrgico, de la conducta anestésica y de las comorbilidades preexistentes. En Francia se informó $47.4 \%$ de las reacciones anafilácticas por BNM. Dentro de ellos succinilcolina (60.6\%), atracurio (19.6\%), cisatracurio (5.9\%), vecuronio (4.6\%), rocuronio (4.3\%), pancuronio (2.7\%) y mivacurio $(1.9 \%)^{(5)}$.

En Australia la variación en la incidencia de anafilaxia por rocuronio es de 1:3,500 a 1:445,000 ${ }^{(1)}$. Se demostró que la tasa de anafilaxia debido a la succinilcolina, rocuronio y atracurio fue de 1:2079, 1:2498 y 1:7680-109,000 respectivamente. Aunque el riesgo de reacciones alérgicas no es la única característica de estos fármacos, los anestesiólogos deben tenerla en cuenta a la hora de hacer su elección clínica. Esto debe ser parte del razonamiento clínico cuando se utilicen estos fármacos ${ }^{(6-8)}$.

El cisatracurio tuvo la menor tasa de reactividad cruzada en pacientes que habían sufrido previamente anafilaxia al rocuronio y vecuronio ${ }^{(2-8)}$, resultados controversiales debido a la dificultad para obtener información ${ }^{(9-12)}$.

Las reacciones alérgicas a los BNM están casi exclusivamente mediadas por IgE. Hasta $75 \%$ de las reacciones se han publicado en el primer contacto conocido con el BNM ${ }^{(13-16)}$. Esto sugiere una posible reacción cruzada con los anticuerpos IgE generados por contacto previo con productos químicos aparentemente no relacionadas.

En general, los síntomas de anafilaxia incluyen varios sistemas de órganos: la piel [principalmente urticaria (80$90 \%)$ ], las vías respiratorias (70\%), tracto gastrointestinal (30-45\%), cardiovasculares (10-45\%) y el sistema nervioso central (10-15) $)^{(17-23)}$.

El grupo amonio cuaternario es responsable del efecto bloqueador neuromuscular como de las propiedades alergénicas de la molécula; esta bivalencia de los BNM explica la liberación de mediadores que ocurre en un paciente sensibilizado, aun en ausencia de proteína transportadora ${ }^{(15,24-27)}$.

Identificar la incidencia de reacciones anafilácticas secundarias al uso de bloqueadores neuromusculares constituye el objetivo de esta investigación.

\section{MATERIAL Y MÉTODOS}

Se realizó una investigación descriptiva, observacional de corte transversal para evaluar la incidencia de reacciones anafilácticas secundarias al uso de BNM. El estudio se realizó en el Servicio de Anestesiología y Reanimación del Hospital «Hermanos Ameijeiras» en La Habana, Cuba durante el período comprendido entre enero de 2016 y diciembre de 2018.

Se consideraron los siguientes criterios de selección:

- Criterios de inclusión: todos los pacientes que en el período perioperatorio presentaran reacciones anafilácticas secundarias al uso de bloqueadores neuromusculares.
- Criterios de exclusión: pacientes con antecedentes personales o familiares de enfermedades neuromusculares, antecedentes de alergia o hipersensibilidad a algunas de las drogas de referencia en el estudio.

- Criterios de salida: cuando los pacientes en el perioperatorio tuvieran complicaciones graves como choque anafiláctico o parada cardíaca que requirieran ser trasladados a la sala de cuidados intensivos.

Muestra: Estuvo constituida por una serie consecutiva de 207 pacientes que cumplieron con los criterios de selección durante el período comprendido entre enero de 2016 y diciembre de 2018.

Descripción de la técnica: todos los pacientes se monitorizaron en el quirófano con un monitor multiparamétrico. Se evaluó la frecuencia cardíaca, presión arterial sistólica y diastólica, electrocardiograma pulsioximetría y capnografía.

Las dosis administradas de BNM fueron acorde a las recomendadas en la literatura. Grupo A (atracurio $0.5 \mathrm{mg} /$ $\mathrm{kg}$ ), grupo $\mathrm{V}$ (vecuronio $0.1 \mathrm{mg} / \mathrm{kg}$ ) y grupo R (rocuronio $0.6 \mathrm{mg} / \mathrm{kg}$ ). Todas por vía endovenosa y a dosis equivalentes.

Una vez iniciada la inducción anestésica se verificó la hora y se evaluaron las posibles complicaciones cada minuto durante $10 \mathrm{~min}$, posteriormente cada $30 \mathrm{~min}$ hasta finalizar el acto quirúrgico. En el postoperatorio inmediato éstas se monitorizaron cada hora en las primeras tres horas.

Análisis estadístico. Para el procesamiento de los datos se utilizó una base de datos en Excel con el empleo del sistema computarizado SPSS versión 16.0. Para cumplir los objetivos propuestos, se analizaron las variables seleccionadas mediante el cálculo de medidas de resumen para variables cualitativas y cuantitativas (números absolutos y porcentajes (\%), media y desviación estándar). Para evaluar la relación entre el tiempo de aparición de la reacción anafiláctica y el grado de complejidad se utilizó el estadístico exacto de Fisher como método de análisis alternativo de la prueba estadística $\chi^{2}$ de Pearson para tablas de $2 \times 2$ cuando tienen una frecuencia esperada inferior a cinco en más de $20 \%$ de sus celdas. Las diferencias fueron consideradas estadísticamente significativas para valores de $\mathrm{p}<0.05$.

Seguridad y ética. El protocolo de investigación fue analizado y aprobado por los profesores del servicio de anestesiología y reanimación del hospital y del consejo científico de la institución. Se tuvieron en cuenta todos los aspectos de seguridad y ética que caracterizan a toda investigación clínica.

\section{RESULTADOS}

Se realizó un total de 7,731 intervenciones quirúrgicas electivas mayores. De ellas, 3,431 pacientes recibieron anestesia general en sus diversas modalidades (general orotraqueal, 
nasotraqueal y combinada) que requirieron el uso de BNM, lo cual constituyó $44.37 \%$ del total.

Con relación a la distribución de las variables biométricas el rango de edades osciló entre 37 y 76 años con una media de $48 \pm 1.6$ años. Predominó el sexo femenino (75\%) de los pacientes. La media del peso de $71.1 \pm 1.5 \mathrm{Kg}$. Prevaleció el riesgo ASA III (49.5\%). Predominaron los pacientes sin trastornos orgánicos, fisiológicos, bioquímicos o psiquiátricos.

Todos los pacientes fueron intervenidos por diversos procedimientos en abdomen superior. La media del tiempo anestésico-quirúrgico fue 226.6 min ( \pm 3.5 horas).

Los BNM utilizados fueron: vecuronio $37 \%$, rocuronio $25 \%$, cisatracurio $6 \%$ y atracurio $27 \%$.

La frecuencia de reacciones adversas según el BNM utilizado fue escasa. Sólo se encontraron cuatro casos: dos por atracurio (50 \%), uno por rocuronio y uno por vecuronio $25 \%$ respectivamente.

En la Tabla 1 se muestran las reacciones adversas según tiempo de aparición, gravedad y tratamiento. El tiempo de aparición de la reacción adversa fue $\leq 10 \mathrm{~s}$ en $25 \%, \leq 30 \mathrm{~s}$ en $25 \%$, menos de un minuto ( $\leq 60 \mathrm{~s}$ ) en dos pacientes $50 \%$.

Las reacciones adversas fueron cuatro en total. El tratamiento en dos pacientes que presentaron urticaria se trató con antihistamínicos y corticoides. En los otros dos que mostraron cuadros asmatiformes fueron broncodilatadores y corticoides. Las cuatro reacciones adversas fueron leves y los síntomas desaparecieron de forma rápida y sin grandes consecuencias.

$75 \%$ tuvo la reacción en el primer contacto con los BNM utilizados.

Estas reacciones se presentaron en dos pacientes por el uso de atracurio, en uno por vecuronio y en otro por rocuronio.

La reacción anafiláctica se mostró una vez terminada la inducción anestésica en 100\% de los pacientes. La reacción principal al atracurio fue erupción cutánea en un paciente y boncoespasmo en otro. Con el rocuronio hubo dolor urente intenso en el sitio de inyección y con el vecuronio un paciente

\begin{tabular}{lll}
\multicolumn{2}{c}{$\begin{array}{c}\text { Tabla 1: Tabla de reacciones adversas según } \\
\text { tiempo de aparición, gravedad y tratamiento. }\end{array}$} \\
& Reacciones adversas & $\mathrm{n}(\%)$ \\
\hline Tiempo de & Inmediato a la inyección $\leq 10 \mathrm{~s}$ & $1(25)$ \\
aparición & Inmediato a la inyección $\leq 30 \mathrm{~s}$ & $1(25)$ \\
Gravedad & Menos de un min & $2(50)$ \\
Tratamiento & Leve & $4(100)$ \\
& Antihistamínicos y corticoides & $2(50)$ \\
Total & Broncodilatadores y corticoides & $2(50)$ \\
\hline
\end{tabular}

Fuente: Hoja de recolección de datos. presentó broncoespasmo. En todos, la severidad del cuadro clínico fue leve y no hubo que lamentar pérdidas de vidas.

\section{DISCUSIÓN}

La mayor parte de los agentes usados en anestesiología se han publicado como responsables de una posible liberación inespecífica de histamina. Dependiendo de la acuciosidad diagnóstica, se ha determinado que entre 8 y 73\% del total a quienes se administró algún BNM, presentaron cierto grado de liberación de histamina ${ }^{(8,12,28)}$.

La identificación del agente es más difícil en las reacciones anafilácticas no alérgicas que en las alérgicas; sin embargo, si se comparan los registros anestésicos de los pacientes, es posible observar ciertas tendencias. El atracurio, clásicamente liberador de histamina, está más relacionado con anafilaxia no alérgica, mientras que el uso de succinilcolina y los BMN aminoesteroides con anafilaxia alérgica ${ }^{(29,30)}$.

Los BNM pueden inducir dos tipos de reacciones: una de tipo inmunológico, IgE dependiente por la estructura del amonio cuaternario $\left(\mathrm{NH}_{4}^{+}\right)$como principal epítope antigénico y otra no inmunológica, especialmente relacionada con el grupo de las bencilisoquinolinas, producto de la estimulación no específica de los mastocitos ${ }^{(31-35)}$.

La mayoría de los estudios concuerdan en que la succinilcolina es el fármaco que produce mayor incidencia relativa de anafilaxia con relación a los otros $\mathrm{BNM}^{(23-25)}$. Por tal razón, no se evaluó en esta serie. Sin embargo, se demostró que las reacciones anafilácticas por el uso de BNM aminoesteroides se presentaron en $67 \%$ de los pacientes (pancuronio 5\%, vecuronio $37 \%$, rocuronio $25 \%$ ), mientras que en los bencilisoquinolineos ocurrió en 33\% (cisatracurio 6\% y atracurio 27\%).

Escolano Villén y colaboradores ${ }^{(11)}$ publicaron en España que los BNM que estaban involucrados en reacciones anafilácticas alérgicas perioperatorias fueron: succinilcolina (33.4\%), rocuronio (29.3\%), atracurio (19.3\%) y vecuronio (10.2\%), hecho que coincide con los hallazgos de esta investigación.

Un grupo importante de autores señalaron ${ }^{(11,15,31,35)}$ que el sexo femenino es el de mayor incidencia. En esta investigación predominó tres veces más en mujeres que en hombres.

Existe una gran discrepancia con relación a la incidencia de reacciones anafilácticas al rocuronio. Mientras en Estados Unidos ocurre en 1/445,000 anestesias y es donde se usa $80 \%$ de la droga que se produce en el mundo, en Francia es considerado un BNM de riesgo elevado y en Noruega se informó en 1/3,500 anestesias ( 55 reacciones y tres muertes en cuatro años), lo que llevó a intervenir a las autoridades de ese país y se retiró de la práctica rutinaria. Su uso está reservado para las intubaciones de urgencia. Los BNM en conjunto ascienden a 93.2\% de las anafilaxias alérgicas en Noruega ${ }^{(2,13,14,32,35)}$.

La incidencia de reacciones no alérgicas a los BNM (no mediadas por IgE) representa entre 20 y 50\% de las reacciones 
adversas $^{(24)}$. Los BNM que liberan histamina son los del grupo bencilisoquinolinas. Hoy en día se utilizan de ese grupo el atracurio y cisatracurio. Aunque este último no libera histamina como el atracurio, su potencial anafiláctico alérgico no es proporcionalmente menor ${ }^{(24-28)}$. El atracurio tiene una incidencia de efectos secundarios graves por liberación de histamina de $1 \%$.

El aparente aumento de la incidencia de anafilaxia al rocuronio en algunos países debe ser interpretado con precaución hasta que se tengan estudios epidemiológicos más numerosos. Es posible que se deba a múltiples factores clínicos, metodológicos y estadísticos que han contribuido a esta aparente contradicción entre ellos ${ }^{(2,8-13)}$.

La reacción anafiláctica se presentó una vez terminada la inducción anestésica en 100\% de los pacientes. La reacción principal al atracurio fue erupción cutánea en un paciente y broncoespasmo en otro. Con el rocuronio hubo dolor urente intenso en el sitio de inyección y con el vecuronio un paciente presentó broncoespasmo. En todos, la severidad del cuadro clínico fue intermedia y no hubo que lamentar pérdidas de vidas.
El único factor predictivo real de una anafilaxia a un BNM es haber tenido una reacción alérgica previa demostrada en uno de ellos, por lo que la única manera de prevenir una reacción alérgica sería evitar el uso de $\mathrm{BNM}^{(11)}$. De este modo, se ha sugerido que las pruebas cutáneas para el diagnóstico de hipersensibilidad al rocuronio y al cisatracurio deben realizarse con concentraciones menores de 1/1,000 y 1/10,000 respectivamente ${ }^{(2,5,34,35)}$.

La mortalidad y la morbilidad secundarias a las reacciones anafilácticas en anestesiología pueden ser evitables si se logra diagnosticarlas y se inicia un tratamiento óptimo precoz.

Se concluye que se constató la presencia de reacciones anafilácticas por el uso de bloqueadores neuromusculares, mismas que se manifestaron en un corto período al inicio de la inducción. El atracurio mostró la mayor frecuencia y todas fueron de intensidad leve.

Conflicto de intereses: Los autores declaran que no existen conflictos de intereses.

\section{REFERENCIAS}

1. Heir PH, Clarke RC, Bunning DL, Platt PR. Anaphylaxis to neuromuscular blocking drugs: incidence and cross-reactivity in Western Australia from 2002 to 2011. Br J Anaesth. 2013;110:981-987.

2. Reddy JI, Cooke PJ, Van Schalkwyk JM, Hannam JA, Fitzharris P, Mitchell SJ. Anaphylaxis is more common with rocuronium and succinylcholine than with atracurium. Anesthesiology 2015;122:39-45.

3. Liew WK, Williamson E, Tang ML. Anaphylaxis fatalities and admissions in Australia. J Allergy Clin Immunol. 2009;123:434-442.

4. Mertes PM, Malinovsky JM, Jouffroy L, Sfa, Aberer W. Reducing the risk of anaphylaxis during anesthesia: 2011 updated guidelines for clinical practice. J Investig Allergol Clin Immunol. 2011;21:442-453.

5. Dong SW, Mertes PM, Petitpain N, Hasdenteufel F, Malinovsky JM. GERAP. Hypersensitivity reactions during anesthesia. Results from the ninth French survey (2005-2007). Minerva Anestesiol 2012;78:868-878.

6. Mertes PM, Volcheck GW. Anaphylaxis to neuromuscular-blocking drugs: all neuromuscular-blocking drugs are not the same. Anesthesiology. 2015;122:5-7.

7. Mertes PM, Tajima K, Regnier-Kimmoun MA, Lambert M, Iohom G, Gueant-Rodriguez RM, et al. Perioperative anaphylaxis. Med Clin NA. 2010;94:761-789.

8. Galvao VR, Giavina-Bianchi P, Castells M. Perioperative anaphylaxis. Curr Allergy Asthma Rep. 2014;14:452.

9. Volcheck GW, Mertes PM. Local and general anesthetics immediate hypersensitivity reactions. Immunol Allergy Clin NA. 2014;34:525-546.

10. Dewachter P, Mouton-Faivre C, Emala CW. Anaphylaxis and anesthesia: controversies and new insights. Anesthesiology. 2009;111:1141-1150.

11. Escolano VF, Sánchez NS. Anafilaxia en anestesia. Rev Esp Anestesiol Rean. 2013;60:55-64.

12. Harper NJ, Dixon T, Dugue P, Edgar DM, Fay A, Gooi HC. Suspected anaphylactic reactions associated with anaesthesia. Anaesthesia. 2009;64:199-211.

13. Kawano T, Tamura T, Hamaguchi M, Yatabe T, Yamashita K, Yokoyama M. Successful management of rocuronium-induced anaphylactic reactions with sugammadex: a case report. J Clin Anesth. 2012;24:62-64.

14. Barthel F, Stojeba N, Lyons G, Biermann C, Diemunsch P. Sugammadex in rocuronium anaphylaxis: dose matters. Br J Anaesth. 2012;109:646-647.
15. Pani N, Dongare PA, Mishra RK. Reversal agents in anaesthesia and critical care. Indian J Anaesth. 2015;59:664-669.

16. Kageyama K, Hayakawa Y, Nakajima Y, Hashimoto S. Anaphylaxis during anesthesia--especially from neuromuscular blocking agents. Masui. 2011;60:55-66.

17. Hagau N, Gherman N, Cocis M, Petrisor C. Antibiotic-induced immediate type hypersensitivity is a risk factor for positive allergy skin tests for neuromuscular blocking agents. Allergol Int. 2016;65:52-55.

18. Rouzaire P, Nosbaum A, Mullet C, Diot N, Dubost R, Bienvenu F et al. Immediate allergic hypersensitivity to quinolones associates with neuromuscular blocking agent sensitization. J Allergy Clin Immunol Pract. 2013;1:273-279.

19. Brusch AM, Clarke RC, Platt PR, Phillips EJ. Exploring the link between pholcodine exposure and neuromuscular blocking agent anaphylaxis. Br J Clin Pharmacol. 2014;78:14-23.

20. Michavila GA, Belver GM, Alvarez NC, Giner MM, Hernando SV, Porto AJ, et al. Drug allergy Work Group of the Spanish Society of Paediatric Allergy, Immunology (GEICAP). Perioperative anaphylactic reactions: Review and procedure protocol in paediatrics. Allergol Immunopathol (Madr). 2015;43:203-214.

21. Dong S, Acouetey DS, Guéant-Rodriguez RM, Zmirou-Navier D, Rémen $\mathrm{T}$, Blanca $\mathrm{M}$, et al. Prevalence of IgE against neuromuscular blocking agents in hairdressers and bakers. Clin Exp Allergy. 2013;43:1256-1262.

22. Michalska-Krzanowska G. Anaphylactic reactions during anaesthesia and the perioperative period. Anaesthesiol Intensive Ther. 2012;44:104111.

23. Mertes PM, De Blay F, Dong S. Allergic risk in anaesthesia. Presse Med. 2013;42:269-79.

24. Mertes PM, Demoly P, Malinovsky JM. Hypersensitivity reactions in the anesthesia setting/allergic reactions to anesthetics. Curr Opin Allergy Clin Immunol. 2012;12:361-368.

25. Blichfeldt L, Garvey LH, Krøigaard M, Mosbech H. Suspected allergic reaction during anesthesia requires thorough postoperative investigation. Ugeskr Laeger. 2012;174:579-580.

26. Ramirez LF, Pereira A, Chiriac AM, Bonnet-Boyer MC, Demoly P. Negative predictive value of skin tests to neuromuscular blocking agents. Allergy. 2012;67:439-441. 
27. Jones PM, Turkstra TP. Mitigation of rocuronium-induced anaphylaxis by sugammadex: the great unknown. Anaesthesia. 2010;65:89-90.

28. Baldo BA, McDonnell NJ, Pham NH. The cyclodextrin sugammadex and anaphylaxis to rocuronium: is rocuronium still potentially allergenic in the inclusion complex form? Mini Rev Med Chem. 2012;12:701-712.

29. Platt PR, Clarke RC, Johnson GH, Sadleir PH. Efficacy of sugammadex in rocuronium-induced or antibiotic-induced anaphylaxis. A case-control study. Anaesthesia. 2015;70:1264-1267.

30. Takazawa T, Tomita Y, Yoshida N, Tomioka A, Horiuchi T, Nagata C, et al. Three suspected cases of sugammadex-induced anaphylactic shock. BMC Anesthesiol. 2014;14:92.
31. McDonnell NJ, Pavy TJ, Green LK, Platt PR. Sugammadex in the management of rocuronium-induced anaphylaxis. Br J Anaesth. 2011;106:199-201.

32. Clarke RC, Sadleir PH, Platt PR. The role of sugammadex in the development and modification of an allergic response to rocuronium: evidence from a cutaneous model. Anaesthesia. 2012;67:266-273.

33. Tsur A, Kalansky A. Hypersensitivity associated with sugammadex administration: a systematic review. Anaesthesia. 2014;69:1251-1257.

34. Baldo BA, McDonnell NJ. Sugammadex and anaphylaxis in the operating theater. Rev Esp Anestesiol Reanim. 2014;61:239-245.

35. Takazawa T, Itsuhata H, Mertes PM. Sugammadex and rocuroniuminduced anaphylaxis. J Anesth. 2015;8:1-8. 